\title{
Role for S. aureus in insulin resistance
}

Infection with Staphylococcus aureus can cause insulin resistance in mice, according to new research published in Nature Microbiology.

$S$. aureus infection is known to occur at increased frequency in some patients with obesity and type 2 diabetes mellitus. In addition, evidence suggests that $S$. aureus disrupts the immune system, which can lead to insulin resistance. However, the mechanistic link between $S$. aureus infection and insulin resistance is unknown, prompting Yu Liu and colleagues to undertake a mouse study to try and elucidate the connection.

The researchers generated a mouse model of S. aureus infection, which exhibited impaired glucose tolerance. A screening assay revealed seven functional extracellular S. aureus proteins. Of these seven proteins, they found that only the extracellular enzymatic domain for lipoteichoic acid synthase (eLtaS), which is released by $S$. aureus, bound to insulin. Using an enzymelinked immunosorbent assay (ELISA), Liu and co-workers were able to show that binding of eLtaS to insulin resulted in insulin being unable to interact with the insulin receptor.
Next, Liu and colleagues created eLtaS transgenic C57BL/6J mice (eLtaS ${ }^{\text {trans }}$ ), which expressed eLtaS without being infected with $S$. aureus and had higher serum levels of triglycerides than wild-type controls. Postprandial hyperglycaemia, which is often associated with the development of type 2 diabetes mellitus and its complications, was higher in the eLtaS $S^{\text {trans }}$ mice than in the control mice, indicating that the transgenic mice had impaired glucose tolerance.

Finally, the researchers developed a human monoclonal antibody against eLtaS (YG2). Administration of YG2 to the transgenic mice or mice challenged with S. aureus restored glucose tolerance. "Our findings reveal that $S$. aureus infection is a risk factor for insulin resistance and provide a potential mechanism underlying insulin resistance," write the authors.

Claire Greenhill

ORIGINAL ARTICLE Liu, Y. et al. The extracellular domain of Staphylococcus aureus $L$ taS binds insulin and induces insulin resistance during infection. Nat. Microbiol. https://doi. org/10.1038/s41564-018-0146-2 (2018)

\section{Sphingolipids involved in T1DM}

New research reports that sphingolipid metabolism is abnormal in the pancreatic islets of patients with type 1 diabetes mellitus (T1DM).

Sphingolipids are known to be involved in $\beta$-cell physiology, with roles in proinsulin folding and insulin section. However, all the evidence to date has been derived from animal models and cell cultures; therefore, Laurits Holm and colleagues investigated sphingolipid metabolism in humans with T1DM.

By immunostaining pancreatic biopsy samples from the DiViD study, the researchers were able to demonstrate that levels of sulfatide (a sphingolipid involved in insulin secretion) were reduced in patients newly diagnosed with T1DM compared with control individuals. Next, they used a microarray analysis to show that the expression of several enzymes involved in sphingolipid metabolism was reduced in patients with T1DM. The researchers also identified polymorphisms in eight genes encoding proteins involved in sphingolipid metabolism that contribute to the genetic predisposition to T1DM.
Finally, the researchers postulated that raising sulfatide levels could be used therapeutically in T1DM. To test this theory, they treated nonobese diabetic (NOD) mice with fenofibrate, a drug that is known to increase sulfatide levels. Following treatment from the age of 3 weeks to 35 weeks, the development of diabetes was prevented in $100 \%$ of the treated mice, compared with $27 \%$ of the control mice. The treated mice showed decreased insulitis and increased expression of sulfatide in islets. Fenofibrate also reversed diabetes in $46 \%$ of treated NOD mice following 3 weeks of treatment.

The authors conclude that upregulating sulfatide biosynthesis could be a promising avenue for developing new therapies for patients with T1DM, and that altering sphingolipid metabolism could be used to prevent and treat T1DM.

\section{Claire Greenhill}

ORIGINAL ARTICLE Holm, L. J. et al. Abnormal islet sphingolipid metabolism in type 1 diabetes. Diabetologia. https://doi.org/10.1007/s00125-018-4614-2 (2018) FURTHER READING Meikle, P. J. \& Summers, S. A. Sphingolipids and phospholipids in insulin resistance and related metabolic disorders. Nat. Rev. Endocrinol. 13, 79-91 (2017)

\section{BAT 'talks' to muscles}

Exercise has many health benefits; however, the underlying mechanisms are largely unknown. Now, new research by Kristin Stanford, Laurie Goodyear and colleagues shows that exercise increases circulating levels of 12,13-dihydroxy-9Z-octadecenoic acid (12,13-diHOME) in humans and mice. This lipokine (a class of lipids released from adipose tissue that act as signalling molecules to influence metabolism) causes skeletal muscles to increase fatty acid oxidation and uptake. The study also showed that brown adipose tissue (BAT) is the source of 12,13-diHOME in mice following exercise.

Whether exercise can regulate lipokine levels was unknown, which prompted the researchers to undertake human and mouse model studies to investigate this issue. Stanford, Goodyear and co-workers recruited 39 healthy young and old, male and female volunteers with different activity levels (from sedentary to active lifestyles). Lipidomics analyses were performed on blood samples from volunteers taken before and after moderately intense exercise. The researchers showed that regardless of sex, age or activity levels, acute exercise substantially increased plasma levels of 12,13-diHOME.

Next, the authors replicated the human study in a mouse model and showed that mice also had elevated levels of 12,13-diHOME after exercise. As previous studies suggested that BAT is a source of 12,13-diHOME, the researchers surgically removed the intrascapular BAT in mice. The exercise-induced increase of 12,13-diHOME was blunted in these mice compared with mice that had sham surgery, which confirms that BAT is a source of 12,13-diHOME.

Finally, the researchers injected mice with a fatty acid and either 12,13-diHOME or a vehicle control. Skeletal muscle uptake of fatty acid was increased in mice that received 12,13-diHOME compared with those that received the control. In addition, cultured mouse muscle fibres incubated with 12,13-diHOME in vitro showed increased mitochondrial respiration.

"Here we see a new function for BAT with exercise, talking to the skeletal muscle to increase metabolism," concludes Goodyear.

Ivone Leong

ORIGINAL ARTICLE Stanford, K. I. et al. 12,13-diHOME: an exercise-induced lipokine that increases skeletal muscle fatty acid uptake. Cell Metab. 27, 1111-1120 (2018) 